\title{
Epigenetic Control of Trefoil Factor Family (TFF) Peptide Expression in Human Retinoblastoma Cell Lines
}

\author{
Claudia Philippeit Maike Busch Nicole Dünker
}

University of Duisburg-Essen, Medical Faculty, Institute of Anatomy, Department of Neuroanatomy, Essen, Germany

\section{Key Words}

Trefoil factor family peptides • Retinoblastoma • Epigenetics • Promoter methylation

\begin{abstract}
Background: Recent studies demonstrated that epigenetic mechanisms are involved in the regulation of trefoil factor family (TFF) peptide expression in cancer. In human tissues with endogenous TFF1, TFF2 or TFF3 gene expression, the corresponding promoter is unmethylated and in organs without TFF expression, the promoter of the three genes is highly methylated. Methods: Retinoblastoma (Rb) cell lines were treated with the DNA methyltransferase inhibitor 5-Aza-2'deoxycytidine (5-Aza-dC), the histone deacetylase inhibitor 4-Phenylbutyric acid (PBA) or both and analyzed for changes (i) in TFF mRNA expression by Real-time PCR and (ii) in the methylation status of the TFF promoters by genomic bisulfite sequencing. Results: The degree of promoter methylation correlates with endogenous TFF expression in the retinoblastoma cell lines analyzed. Nearly all Rb cell lines exhibiting high endogenous TFF1 expression displayed low methylation of the CpGs in the corresponding promoter region. Low expression of TFF3 in $\mathrm{Rb}$ cell lines is linked with high density methylation of the TFF3 promoter. 5-Aza-dC treatment induced TFF1 and TFF3 expression in nearly all cell lines investigated and combined treatment with PBA further increased this effect. The number of methylated $\mathrm{CpG}$ dinucleotides of the TFF promoter is clearly reduced upon treatment with 5-Aza-dC and combined treatment with PBA further extended the degree of demethylation. Conclusion: Our data clearly show that the expression of TFF3 in retinoblastoma cell lines is epigenetically regulated, whereas the level of TFF1 and TFF2 seems to be regulated by other or additional mechanisms.
\end{abstract}

Copyright @ 2014 S. Karger AG, Basel

Prof. Dr. Nicole Dünker
University of Duisburg-Essen, Medical Faculty, Institute of Anatomy, Department of Neuroanatomy, Hufelandstr. 55, 45122 Essen (Germany) Tel. +49-201-723-4299, Fax +49-201-723-5635, E-Mail nicole.duenker@uk-essen.de 


\section{Introduction}

Trefoil factor family (TFF) peptides are members of a protein family characterized by a three-loop structure formed by a distinct motif composed of cysteine disulfide bonds. The three known TFF peptides, TFF1 (formerly pS2), TFF2 (formerly spasmolytic protein; SP) and TFF3 (formerly intestinal trefoil factor, ITF) are small, 6.5-14 kDa proteins, which are often secreted together with mucines (for review: [1-3]).

TFF peptides are major constituents of the mucosa, with TFF1 and TFF2 being expressed in gastric epithelia and TFF3 expression was detectable in all tissues containing mucussecreting cells [3]. Besides their prominent expression in mucous epithelia, TFF peptides are also synthesized in the central nervous system of rodents [4-7]. TFF1 was found to be uniformly expressed in all brain regions, whereas TFF3 expression was limited to the hippocampus, the temporal cortex and the cerebellum. TFF3 mRNA was found mainly in neurons and not in glia cells [7]. TFF1 and TFF3 mRNA expression has also been detected in healthy human cornea, whereas only TFF3 was immunohistochemically detected in different corneal diseases [8] and a function of TFF3 in corneal wound re-epithelialization was demonstrated [9].

Next to their expression in normal tissues, TFF expression has been described in many cancer cell lines [10-13]. In the current literature, TFF peptides are discussed as tumor suppressors and potential tumor progression or oncogenic factors [14]. On the one hand, TFF peptides are overexpressed in several human solid tumors [15-17]. TFF3 overexpression is frequently observed in human cancers e.g. gastric cancer [18], pancreatic cancer [19], hepatocellular carcinomas [20], colon carcinoma [21] and breast cancer [13, 22]. Besides, TFF3 expression correlates with the tumor grade in hepatocellular carcinoma [23] and TFF3 is highly expressed in intestinal metaplasia and a marker for poor prognosis in gastric carcinoma [24]. On the other hand, TFF1-knockout mice develop gastric carcinomas and adenomas [25] and patients with gastric cancer usually display reduced TFF1 levels. Thus, TFF1 has been described as a tumor suppressor gene in gastric carcinoma [26]. Besides, a deficiency in TFF1 increases the tumorigenicity of human breast cancer cells, supporting a tumor suppressor role [27].

Epigenetic alterations, resulting in site-specific DNA methylation and histone deacetylation, are well-known mechanisms associated with transcriptional silencing of cancer-related genes [28-31]. DNA methylation within the genome of vertebrates occurs at cytosines located $5^{\prime}$ to a guanosine, called $\mathrm{CpG}$ dinucleotide. Short regions, known as CpG islands, are rich in $\mathrm{CpG}$ content and frequently found in the proximal promoter region of many human genes (for review: see [29, 30, 32]). In early studies in 1993, Antequera and Bird [33] estimated that about $60 \%$ of the known human genes are associated with CpG islands, which are mostly unmethylated. They concluded that $\mathrm{CpG}$ islands could be free of methylation even when the corresponding gene is silent, because of the tissue-restricted pattern of some genes [32, 33].

DNA-binding transcription factors recognize a specific sequence in the promoter region and cause transcriptional activation or repression of the related gene [29]. Methyl-CpG binding proteins serve as transcriptional repressors rendering methylated DNA inaccessible to transcription factors through histone modifications and changes in chromatin structure [34]. Dense methylation of DNA has been associated with transcriptional repression of chromatin characterized by under-acetylated histones [35]. On the other hand, histone acetyltransferases acetylate histones and cause a weakening of the bonds between histones and DNA, promoting exposure of DNA to the transcriptional machinery [36]. Thus, independent of alterations in the DNA sequence, epigenetic mechanisms enable transient response to environmental variance through changes in gene expression [34].

Little is known about the role of DNA methylation and histone deacetylation in transcriptional inhibition of tumor suppressor genes inactivated in cancer cells in general and about their role in the regulation of TFF gene expression in retinoblastoma cells in particular. The three TFF genes are tandemly clustered within a $50 \mathrm{~kb}$ sequence on 
chromosome 21q22.3 [37-39] and recent studies demonstrated that all TFFs contain CpG dinucleotides in their promoter region [40], although with lower CpG densities than in classically defined CpG islands [41]. Besides, cell-specific TFF gene expression seems to be transcriptionally regulated through motifs located upstream of the TATAA box [42]. In organs without detectable TFF content the promoter region is methylated, but in tissues with endogenous TFF gene expression the related promoter is free of methylation [43].

Epigenetic mechanisms are also involved in the regulation of TFF expression in cancer. A most recent study in 2014 by Feng et al. demonstrated that the methylation status of the CpG islands in the promoter region correlate with TFF1 expression levels in human gastric cancer cells and DNA methylation is a key mechanism of silencing TFF1 in gastric carcinomas [26]. Besides, TFF1 has been described as a differentially methylated gene in Rb tumors [44] and as one of the up-regulated genes in primary retinoblastomas with a matching activating histone modification [45].

In the present study we set out to determine if the expression of TFF genes is epigenetically regulated in different retinoblastoma cell lines.

\section{Materials and Methods}

\section{Cell culture}

The 8 human retinoblastoma (Rb) cell lines RBL-13, RBL-15, RBL-30, RB 247 C3, RB 355, RB 383, WERI-Rb1 and Y-79 [46-49] were cultivated as suspension cultures in Dulbecco's modified Eagle's medium (DMEM; PAN-Biotech) with $10 \%$ fetal calf serum (FCS; PAN-Biotech), $100 \mathrm{U}$ penicillin/ml and $100 \mu \mathrm{g}$ streptomycin/ml (Invitrogen), $4 \mathrm{mM}$ L-glutamine (Sigma), $50 \mu \mathrm{M} \beta$-mercaptoethanol (Roth) and $10 \mu \mathrm{g}$ insulin $/ \mathrm{ml}$ (Sigma) at $37^{\circ} \mathrm{C}, 10 \% \mathrm{CO}_{2}$ and $95 \%$ humidity.

\section{Epigenetic drug treatment}

For epigenetic drug treatment 1-1.5 x 106 Rb cells were seeded on poly-D-lysine (Sigma) coated 6-well plates and cultivated in $3 \mathrm{ml}$ complete DMEM. Rb cell lines were either treated for $48 \mathrm{~h}$ with the DNAdemethylating agent 5-Aza-2 deoxycytidine (5-Aza-dC, $1 \mu \mathrm{M}$; Sigma-Aldrich) dissolved in DMSO prior to 5 days recovery or were treated continuously for 7 days with $1 \mathrm{mM}$ 4-Phenylbutyric acid (PBA; SigmaAldrich) also dissolved in DMSO. A combination of both inhibitors was also given to all cell lines under the same conditions. Control cells (untreated solvent control) were grown in parallel and received equivalent amounts of DMSO (i) for $48 \mathrm{~h}$ prior to 5 daily medium changes without DMSO (5-Aza-dC control), (ii) continuously for 7 days (PBA control) or (iii) for 2 days (DMSO double dose) followed by 5 days DMSO single dose (5-Aza-dC + PBA control), resulting in three individual control groups. Growth medium was changed every $24 \mathrm{~h}$ and cells were harvested on day 7 according to a protocol published before by Vestergaard et al. [40]. RNA and DNA were extracted from these cells using the protocols described below. All experiments were performed in triplicate and repeated twice.

\section{Genomic DNA preparation and bisulfite sequencing}

Genomic DNA from all Rb cell lines, healthy human donor retinas (kindly provided by S. Thanos) and samples of human duodenum (kind gift of E. Cario) was isolated using the DNeasy Blood and Tissue Kit (Qiagen) and bisulfite-converted with the EZ DNA Methylation-Gold Kit (Zymo Research) to convert all cytosines to uracil, while the methylated cytosines remain unmodified. TFF1, TFF2 and TFF3 promoter sequences were amplified from bisulfite-converted DNA by PCR using the following protocol: $5 \mathrm{~min}$ at $95{ }^{\circ} \mathrm{C}, 50$ cycles of $1 \min 95^{\circ} \mathrm{C}, 2 \min 53 / 54^{\circ} \mathrm{C}, 1 \min 72^{\circ} \mathrm{C}$ followed by final $10 \min 72^{\circ} \mathrm{C}$ step. PCR forward and reverse primer sequences were designed according to a protocol published before [40] and were as follows: TFF $1_{\text {for }}$ GGAATGGGTTTTATGAGTTTTTTT, TFF1 $1_{\text {rev }}$ CATTACCTCCTCTCTACTCCAAAAA; TFF2 $_{\text {for }} \quad$ GGTGTGATTTTGTGTGTGTTTAGT, TFF2 $2_{\text {rev }}$ AAAACCCTCTCCTTCACTTACAAAA; TFF3 $3_{\text {for }}$ AGGAAAGATAAGGAATTTTTGTGTTTT, TFF3 ${ }_{\text {rev }}$ ACATACCTTTATCAAACTCCCAAAC. Afterwards, the PCR product was purified from agarose gels by Illustra GFX PCR DNA and Gel Band Purification Kit (VWR Lifescience) and subcloned into the $\mathrm{pCR}^{\circledR} 4-\mathrm{TOPO}^{\circledR}$ vector using a TOPO TA Cloning Kit for Sequencing (Invitrogen). Plasmid isolation was performed with the GeneJET Plasmid Miniprep Kit (Thermo Scientific) 
Philippeit/Busch/Dünker: Epigenetic Regulation of TFFs in Rb Cells

and individual clones were sequenced by Microsynth using the M13 reverse primer. Sequences of at least 5 clones were analyzed using the Clone Manager 9 software to identify methylated cytosine residues.

\section{RNA isolation and semi-quantitative RT-PCR}

Isolation of total RNA from Rb cell lines was performed using the NucleoSpin RNA II kit (Machery and Nagel) following the manufacturer's protocol. Human total retina RNA from a pool of 6 female/male Caucasians was purchased from Clontech (cat.\# 636579). Besides, normal human duodenum (kindly provided by R. Doliva) was used as a positive control for TFF gene expression. Equal amounts of RNA were subjected to a reverse transcription (RT) reaction using the QuantiTect Reverse Transcription Kit (Quiagen) following the manufacturer's instructions. $2.5 \mu \mathrm{l}$ of the RT-reaction product were used in $50 \mu \mathrm{l}$ PCR reactions with the following specific primer pairs: TFF $1_{\text {for }}$ GGTCGCCTTTGGAGCAGAG, TFF $1_{\text {rev }}$ GCAGCCCTTATTTGCACAC [50]; TFF2 $2_{\text {for }}$ CACCGAATTCTGCAGCTGAGCTAGACATGG, TFF2 $2_{\text {rev }}$ AACCCTCGAGAACACCCGGTGAGCCAGATG; TFF3 $_{\text {for }}$ GTGGTCATGGCTGCCAGAG, TFF3 $3_{\text {rev }}$ AGCTGGAGGTGCCTCAGAAG. PCR products were amplified using the following touch-down PCR program: $5 \mathrm{~min}$ at $95^{\circ} \mathrm{C}, 5$ cycles of $30 \sec 95^{\circ} \mathrm{C}, 15 \sec 61^{\circ} \mathrm{C}, 30 \sec 72^{\circ} \mathrm{C}$, 5 cycles of $30 \sec 95^{\circ} \mathrm{C}, 15 \sec 60^{\circ} \mathrm{C}, 30 \sec 72^{\circ} \mathrm{C}, 5$ cycles of $30 \sec 95^{\circ} \mathrm{C}, 15 \sec 59^{\circ} \mathrm{C}, 30 \sec 72^{\circ} \mathrm{C}$ and 20 cycles of $30 \sec 95^{\circ} \mathrm{C}, 15 \sec 58^{\circ} \mathrm{C}, 30 \sec 72^{\circ} \mathrm{C}$ followed by final $10 \min 72^{\circ} \mathrm{C}$ step. GAPDH was used as an endogenous control with the following conditions: $5 \mathrm{~min}$ at $95^{\circ} \mathrm{C}, 25$ cycles of $30 \mathrm{sec}$ at $95^{\circ} \mathrm{C}, 15 \mathrm{sec}$ at 59 ${ }^{\circ} \mathrm{C}$ and $30 \mathrm{sec}$ at $72{ }^{\circ} \mathrm{C}$ followed by a last amplification step $5 \mathrm{~min}$ at $72^{\circ} \mathrm{C}$. GAPDH primers were $\mathrm{GAPDH}_{\text {for }}$ CATCACCATCTTCCAGGAGC; GAPDH ${ }_{\text {rev }}$ ATGACCTTGCCCACAGCCTT. PCR products were separated on $2 \%$ agarose gels and stained with GelRed Nucleic Acid Stain (Biotium).

\section{Quantitative Real-time PCR}

Quantitative Real-time PCR analyses were performed using a 7300 Real-time PCR System (Applied Biosystems). The following TaqMan ${ }^{\circledast}$ Gene Expression Assays (Applied Biosystems) were used: TFF1 (ID Hs00907239_m1), TFF2 (ID Hs00193719_m1) and TFF3 (ID Hs00902278_m1). GAPDH (ID Hs99999905_ m1) was used as an endogenous control. Real-time PCR reactions using the TaqMan Universal PCR Master Mix (Applied Biosystems) were performed in duplicate and a total volume of $20 \mu \mathrm{l}$ was applied to the following program: $2 \min 50^{\circ} \mathrm{C}, 10 \min 95^{\circ} \mathrm{C}$ followed by 40 cycles of $15 \sec 95^{\circ} \mathrm{C}$ and $60 \sec 60^{\circ} \mathrm{C}$. Relative quantification was calculated by the 7300 Real-time PCR System software (Applied Biosystems).

\section{Statistical analysis and ethical statement}

All experiments were performed in triplicate. Data are means \pm SEM and represent at least two to three independent experiments. Data were analyzed by paired Student's $t$ test and considered significantly different if $* p<0.05$, $^{* *} \mathrm{p}<0.01$, or ${ }^{* * *} \mathrm{p}<0.005$. The research reported was conducted in accordance with the ethical standards established by the United States National Institute. All investigations followed the tenets of the Declaration of Helsinki and were approved by the institutional human experimentation committee.

\section{Results}

Endogenous expression levels of TFF1 and TFF3 are diametrically opposed in human retina and retinoblastoma cell lines

To investigate and compare TFF expression patterns in retina and retinoblastoma cell lines, total RNA was isolated and analyzed by RT-PCR and quantitative Real-time PCR. The specificity of the TFF RT-PCR primer pairs was tested using human duodenum (Fig. 1), shown to be an appropriate positive control for all three TFF genes $[51,52]$. When we compared the RNA levels of the three TFFs in the healthy human retina, we found low expression of TFF1 and high levels of TFF3, while TFF2 is not detectable (Fig. 2).

Compared to the human retina, TFF1 is up-regulated in all $\mathrm{Rb}$ cell lines investigated except in RB 355 and Y-79 Rb cells (Fig. 2A). These results have already been confirmed by Real-time PCR and Western Blot in a previous paper by our group [50]. By contrast, no TFF2 (Fig. 2B) and only trace amounts of TFF3 (Fig. 2C) were detectable in all Rb cell lines. For TFF1 and TFF3, these semi-quantitative RT-PCR results were confirmed by quantitative Real-time PCR data (Fig. 2A, 2C). 
Fig. 1. Specificity of the three TFF primer pairs as revealed by semi-quantitative RT-PCR using human duodenum cDNA as a positive control. Contamination and unspecific binding were excluded using a sterile water reaction as a negative control. The 100 bp molecular ladder standard is shown on the left. TFF transcript sizes are indicated on the right.

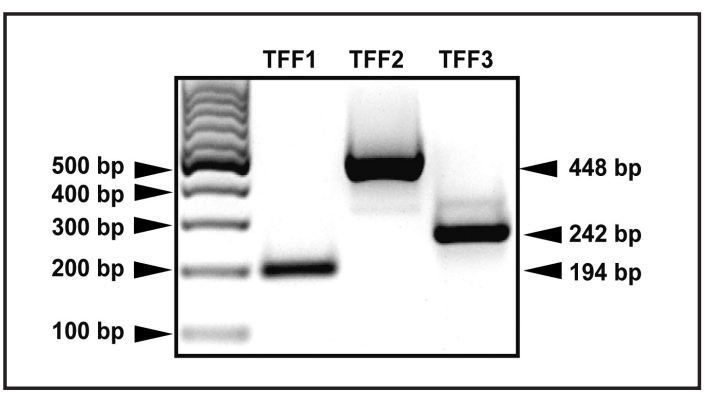

Fig. 2. Endogenous expression of TFF1 (A), TFF2 (B) and TFF3 (C) in the healthy human retina, eight retinoblastoma cell lines and human duodenum as revealed by semi-quantitative RT-PCR and Real-time PCR. The integrity of the cDNAs was tested by amplification of the GAPDH transcript. Healthy human retina was used as a reference and set as 1 . The healthy human retina exhibited low expression of TFF1 and high levels of TFF3, while TFF2 was not detectable. TFF1 is up-regulated in nearly all $\mathrm{Rb}$ cell lines investigated, while no TFF2 and only trace amounts of TFF3 were detectable. Data are means \pm SEM from 3 independent assays. $* \mathrm{p}<0.05$ or ${ }^{* * *} \mathrm{p}<0.005$ statistical differences compared to the healthy human retina pool calculated by paired Student's $t$ test.

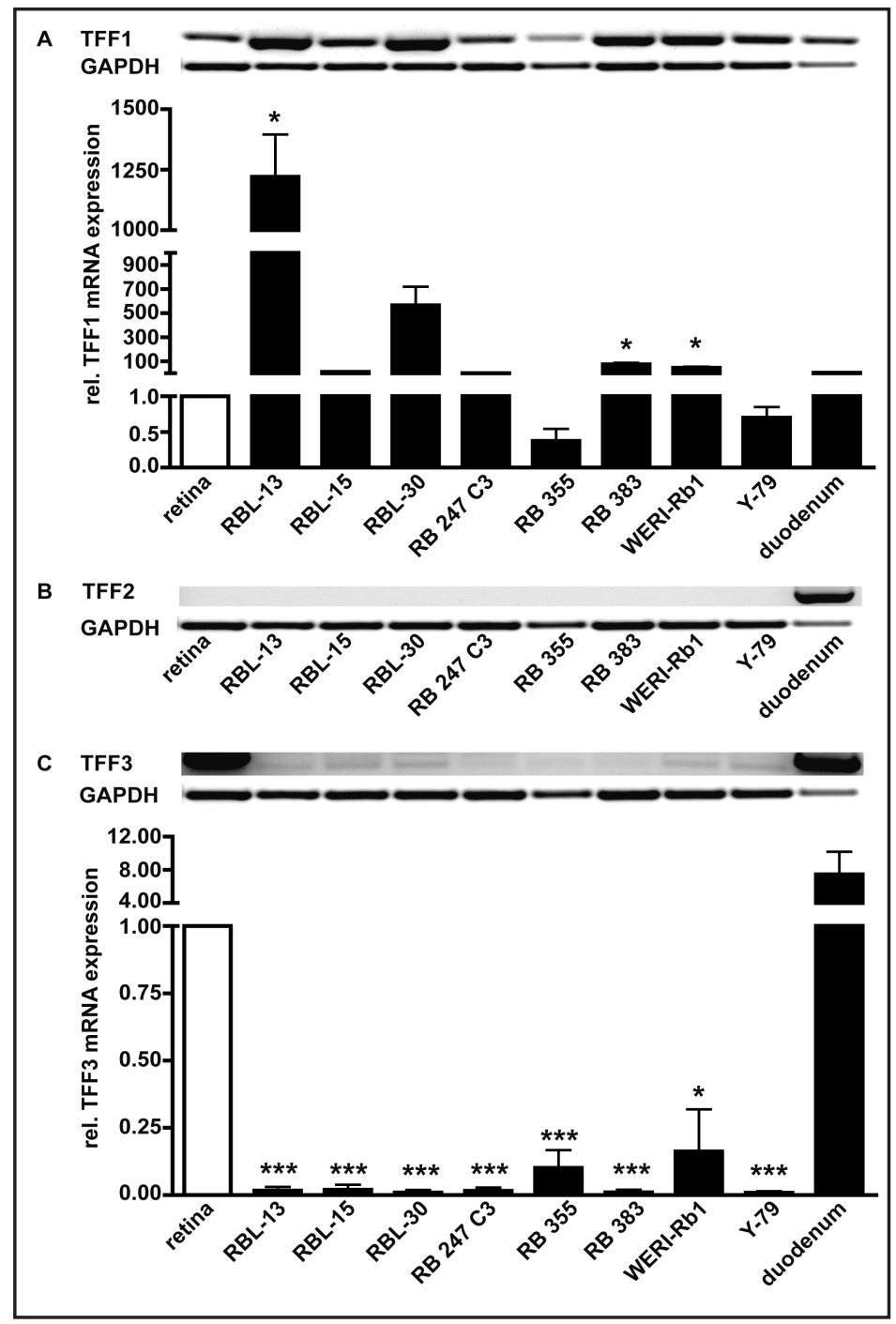

TFF promoter methylation correlates with the endogenous TFF gene expression in retinoblastoma cell lines

To investigate a potential epigenetic regulation of trefoil factor family peptide gene expression in retinoblastoma cell lines, TFF promoter methylation was analyzed by genomic bisulfite sequencing. On the basis of a study by Vestergaard et al. [40], investigating the promoter methylation of TFF peptides in prostate cancer, a total of 13 CpGs in the TFF1 promoter, $16 \mathrm{CpGs}$ in the TFF2 promoter and $8 \mathrm{CpGs}$ in the TFF3 promoter of 8 retinoblastoma cell lines were examined (Fig. 3) and compared to the TFF promoter methylation status of a healthy human retina pool (4 combined retina specimen) and a healthy human duodenum 


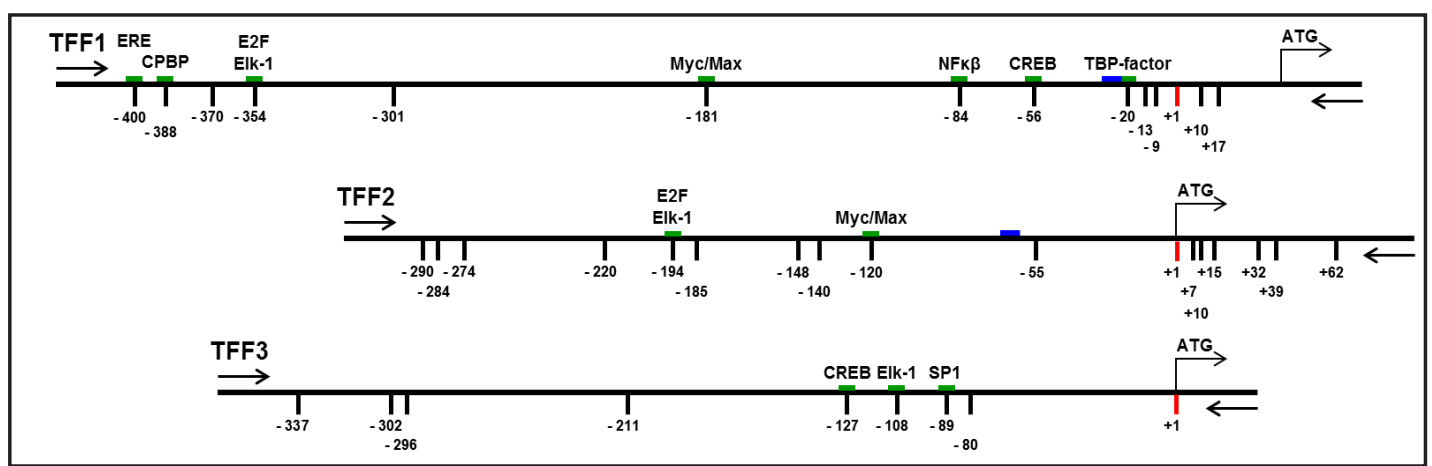

Fig. 3. Schematic illustration of the promoter region of the three TFF genes modified according to [40]. The location of the PCR primer pairs are indicated by arrows. The promoter associated CpG dinucleotides are marked by vertical bars. Nucleotide positions relative to the translation start site (+1) are given; for TFF1 the positions are indicated as published in Jeltsch et al. [63]. Blue bars (-) above the promoter sequence represent the TATAA Box [64]. Putative binding sites for transcription factors in silico predicted by MatInspector 2.6 software are shown in green.

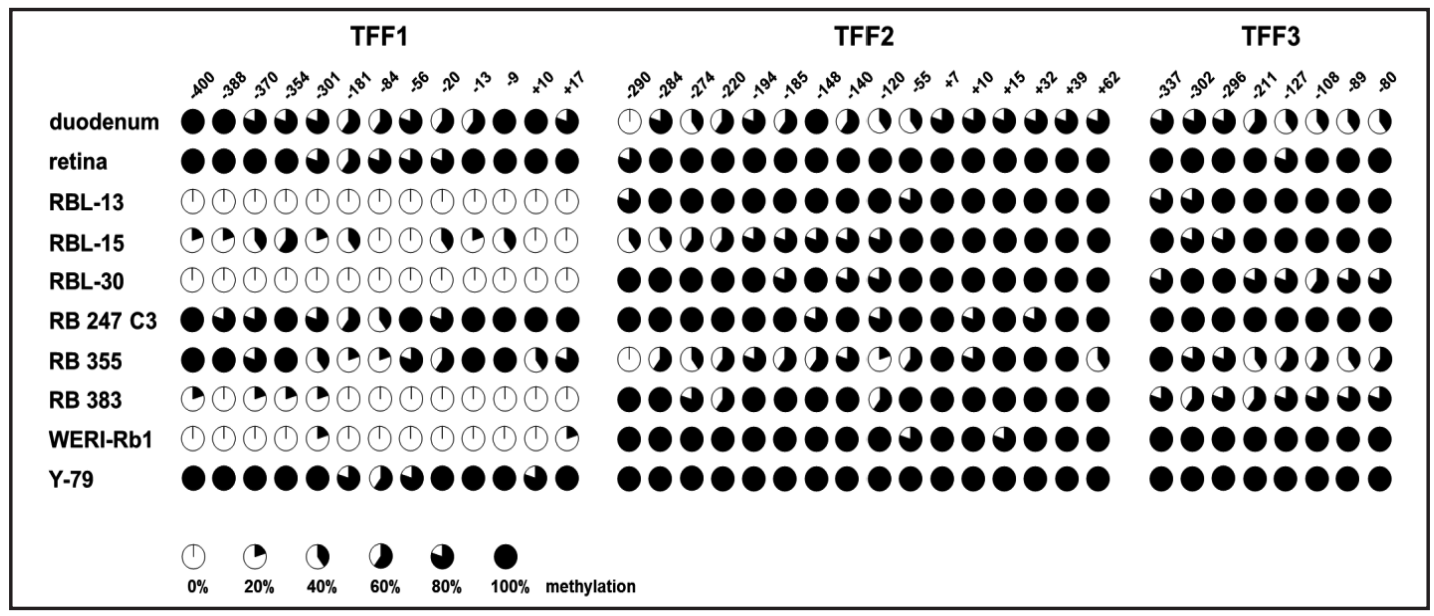

Fig. 4. Genomic bisulfite sequencing of the TFF1, TFF2 and TFF3 promoter associated CpG sites in eight retinoblastoma cell lines. Promoter methylation of human duodenum and healthy human retina were used as a reference. Each circle represents a single $\mathrm{CpG}$ site. The average methylation of all $\mathrm{CpG}$ dinucleotides analyzed is indicated based on sequencing of at least five clones. The TFF promoter methylation correlated with the endogenous TFF gene expression in retinoblastoma cell lines. Nearly all CpGs in the TFF1 promoter of the healthy human retina, human duodenum and $\mathrm{Rb}$ cell lines exhibiting low endogenous TFF1 expression were methylated. By contrast, in Rb cell lines with high to moderate endogenous TFF1 levels all CpGs were completely free of methylation or highly demethylated. In the duodenum reference, exhibiting high endogenous TFF3 expression, nearly all CpGs were somehow demethylated, whereas nearly all Rb cell lines showing only trace amounts of endogenous TFF3 displayed high density methylation of all CpGs. Only minor differences in the methylation patterns of the TFF2 promoter were observed between retinoblastoma cell lines and the healthy human retina.

pool (4 different samples) as a reference (Fig. 4). The degree of cytosine conversion upon sodium bisulfite treatment ranged between $96 \%$ and $100 \%$ (data not shown).

Nearly all CpGs in the TFF1 promoter of the healthy human retina and human duodenum with low endogenous TFF1 expression were methylated (60 \% - $100 \%$; Fig. 4). RB 355 and Y-79 Rb cell lines exhibiting low endogenous TFF1 expression also displayed a high methylation status of nearly all CpGs investigated. By contrast, in Rb cell lines with high to moderate endogenous TFF1 levels all CpGs were completely free of methylation 
(RBL-13, RBL-30) or highly demethylated to a total methylation level of $\leq 23 \%$ (RBL-15, RB 383, WERI-Rb1; Fig. 4; Tab. 1). Thus, TFF1 expression levels seemed to be epigenetically regulated in these retinoblastoma cell lines. The Rb cell line RB 247 C3 represented an exception with a high methylation status, comparable to RB 355 or Y-79, and elevated endogenous TFF1 expression.

Only minor differences in the methylation patterns of the TFF2 promoter were observed between retinoblastoma cell lines and the healthy human retina (Fig. 4). All but two Rb cell lines (RBL-15, RB 355) displayed high levels of hypermethylation of the $16 \mathrm{CpGs}$ investigated, matching the lack of endogenous TFF2 expression. Although RBL-15 and RB 355 cells exhibited a lower methylation level of 81 $\%$ and $65 \%$, respectively (Tab. 1), resembling the methylation status of the human duodenum
Table 1. Methylation status of the TFF promoter region in human duodenum, human retina and eight retinoblastoma cell lines calculated based on genomic bisulfite sequencing. Data indicate the average of $\mathrm{CpG}$ methylation

\begin{tabular}{lccc}
\hline \multirow{2}{*}{ sample } & \multicolumn{3}{c}{ methylation status [\%] } \\
& of the TFF promoter regions \\
& TFF1 & TFF2 & TFF3 \\
\hline duodenum & 80 & 65 & 58 \\
retina & 91 & 99 & 98 \\
RBL-13 & 0 & 98 & 95 \\
RBL-15 & 23 & 81 & 95 \\
RBL-30 & 0 & 96 & 83 \\
RB 247 C3 & 86 & 95 & 100 \\
RB 355 & 71 & 65 & 65 \\
RB 383 & 6 & 94 & 75 \\
WERI-Rb1 & 3 & 98 & 100 \\
Y-79 & 92 & 100 & 100 \\
\hline
\end{tabular}

reference (65\% total methylation) with strong endogenous TFF2 expression, these Rb cell lines still displayed no detectable TFF2 expression, suggesting, that TFF2 expression is not epigenetically regulated.

In the TFF3 promoter region of the healthy human retina the $8 \mathrm{CpGs}$ were almost completely methylated except for CpG -127 (Fig. 4). In the duodenum reference, exhibiting high endogenous TFF3 expression, all CpGs analyzed were hypomethylated to a total methylation degree of $58 \%$ (Tab. 1). By contrast, nearly all Rb cell lines (except RBL-30, RB 355 and RB 383) showing only trace amounts of endogenous TFF3 displayed high density methylation of all CpGs, suggesting an epigenetic regulation of TFF3 expression levels in retinoblastoma cell lines.

Several of the CpGs in the TFF1 and TFF3 promoter correspond with putative binding sites for cellular transcription factors (Fig. 3), as identified by MatInspector 2.6 software analysis [53]. In the TFF1 promoter region of the Rb cell lines analyzed, we monitored differences in the methylation status of (i) CpG -400 overlapping the transcription factor binding site for the estrogene responsive element (ERE), (ii) CpG -388 representing the binding site for the core promoter-binding protein (CPBP) as well as (iii) CpG -354, matching the E2F and the human ETS1 factor (Elk-1) binding site. Besides, in the TFF3 promoter, we observed that the methylation status of CpG -127, matching the binding site of the cAMPresponsive element binding protein (CREB), varies between Rb samples with different TFF3 expression. Notably, these putative binding sites for transcription factors have been shown to play a role in the regulation of cell survival, cell cycle control and proliferation processes, in which TFFs are involved.

Treatment with 5-Aza-2'deoxycytidine and 4-Phenylbutyric acid induce an up-regulation of TFF1 and TFF3 expression in retinoblastoma cell lines

In order to functionally prove the hypothesis that TFF gene expression in retinoblastoma cell lines is indeed epigenetically regulated, we tested the influence of DNA methylation and histone acetylation. For this purpose, cells were treated with the DNA methyltransferase inhibitor 5-Aza-2 deoxycytidine (5-Aza-dC), the histonedeacetylase inhibitor 4-Phenylbutyric acid (PBA) or both and the expression of TFF1, TFF2 and TFF3 was analyzed by Real-time PCR.

Upon application of 5-Aza-dC, in most Rb cell lines TFF1 expression only increased to a low extent, with the exception of RB 355, being the only the semi-adherent cell line 
Fig. 5. Normalized TFF1 (A) and TFF3 (B) mRNA expression in eight retinoblastoma cell lines after treatment with 4-Phenylbutyric acid (PBA) or 5-Aza-2`deoxycytidine (5-Aza-dC) or both as revealed by Real-time PCR. Control cells were treated in parallel with equivalent amounts of DMSO (for details see "material and methods") and set as 1 to normalize mRNA expression. Treatment of retinoblastoma cell lines with the histone deacetylase inhibitor PBA slightly down-regulated TFF1 mRNA levels in some $\mathrm{Rb}$ cell lines, but significantly increased TFF3 mRNA expression in other cell lines. 5-Aza$\mathrm{dC}$ treatment lead to a significant up-regulation of TFF1 and TFF3 ex-

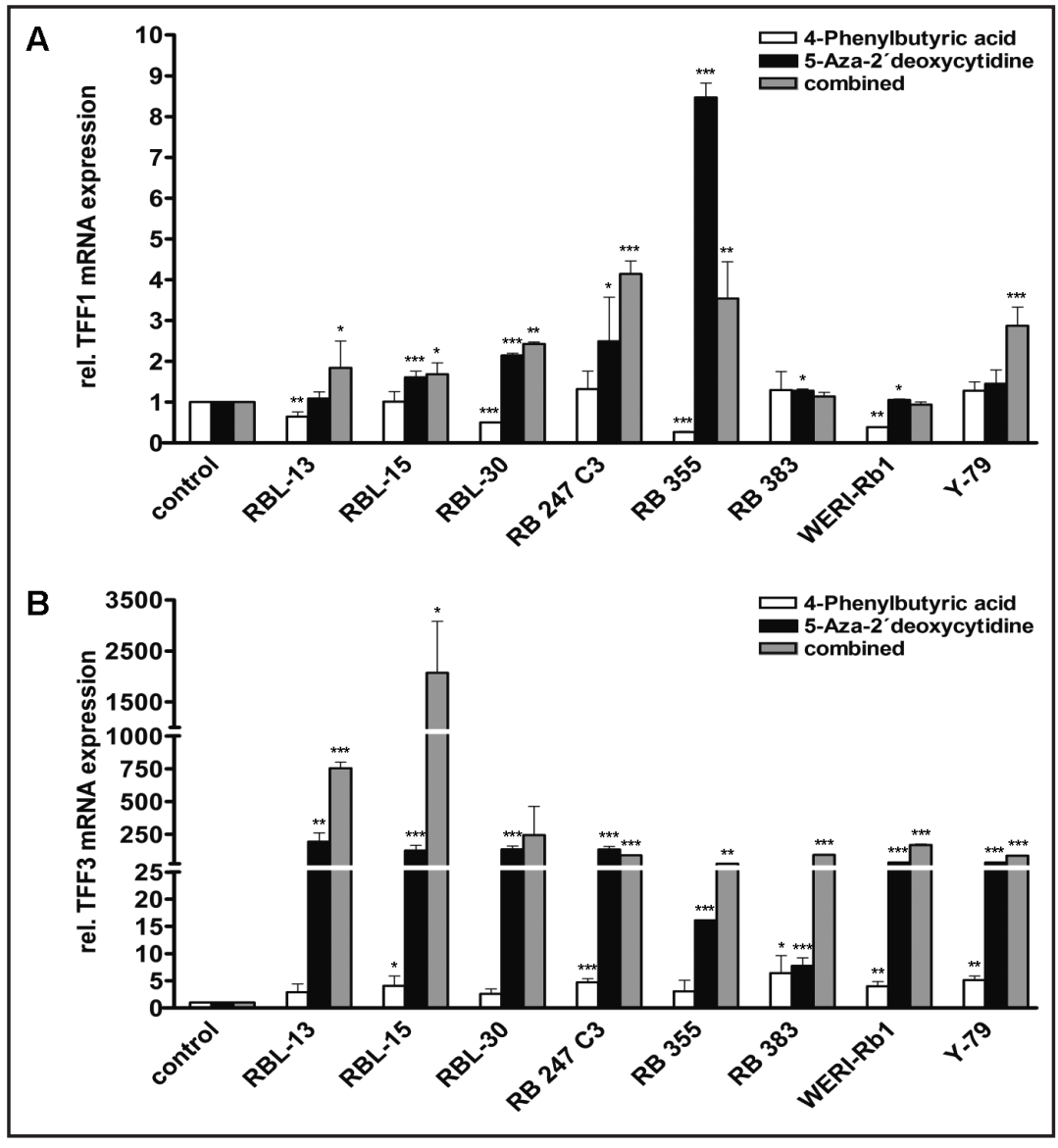

pression in nearly all cell lines investigated and the combined drug treatment effectively induced further changes in the TFF1 and TFF3 content. All experiments were carried out in triplicate and repeated twice. Error bars represent \pm SEM. $* \mathrm{p}<0.05$, ** $\mathrm{p}<0.01$ or $* * * \mathrm{p}<0.005$ statistical differences compared to the control group calculated by paired Student's $t$ test.

investigated and displaying an unexpected high up-regulation of TFF1 gene expression (Fig. 5A). 5-Aza-dC treatment, however, significantly induced TFF3 expression in all cell lines with low and absent endogenous TFF3 mRNA levels, e.g. up to 193-fold in RBL-13 cells (Fig. 5B), suggesting that the induction is dependent on basal endogenous expression levels (Fig. 2).

Treatment of the retinoblastoma cell lines with the histone deacetylase inhibitor PBA induced an unexpected significant down-regulation of TFF1 in 4 out of $8 \mathrm{Rb}$ cell lines investigated (RBL-13, RBL-30, RB 355 and WERI-Rb1; Fig. 5A). By contrast, TFF3 mRNA expression in RBL-15, RB 247 C3, RB 383, WERI-Rb1 and Y-79 cells significantly increased upon PBA treatment (Fig. 5B). However, the 2.6-fold (RBL-30) to 6.4-fold (RB 383) increase in TFF3 expression observed is relatively low compared to the induction obtained by 5-AzadC treatment.

The combined drug treatment effectively induced a significant up-regulation of TFF1 mRNA expression in all Rb cell lines investigated except in RB 383 and WERI-Rb1 cells (Fig. $5 \mathrm{~A}$ ). Compared to the significant induction of TFF3 expression upon 5-Aza-dC, TFF3 levels of all cell lines investigated, except RBL-30 and RB 247 C3, further increased upon 5-Aza-dC/ PBA double treatment (Fig. 5B).

No induction of TFF2 expression was found upon treatment with 5-Aza-dC, PBA or both inhibitors (data not shown). 


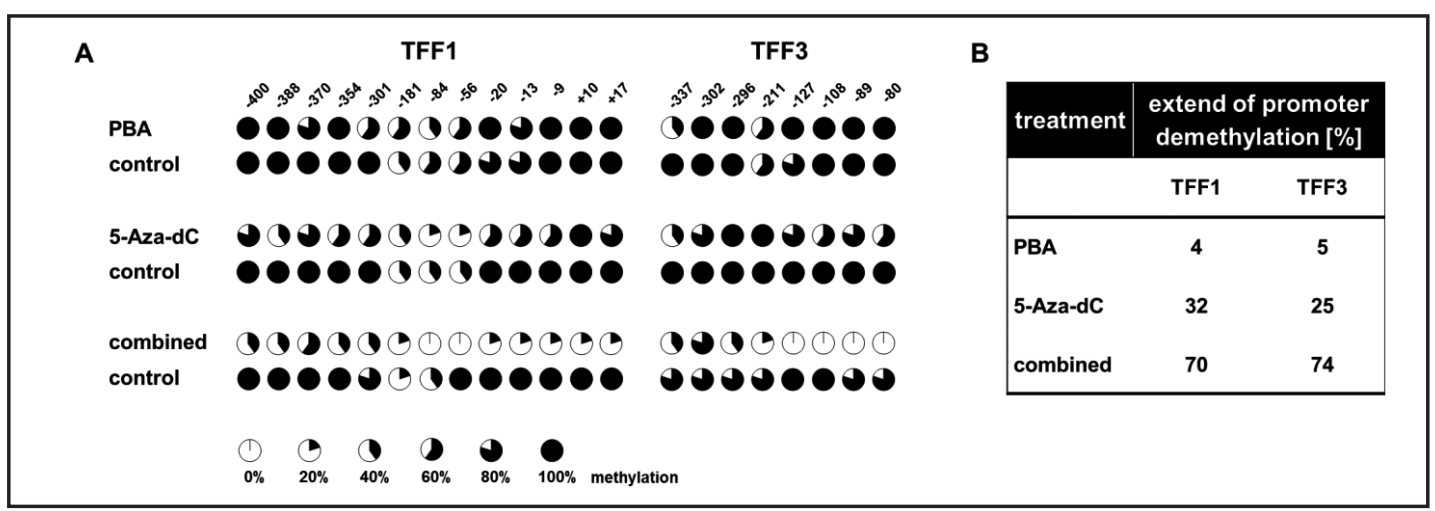

Fig. 6. (A) Genomic bisulfite sequencing of the TFF1 and TFF3 promoter in the retinoblastoma cell line Y-79 following treatment with the histone deacetylase inhibitor PBA or the DNA methyltransferase inhibitor 5-Aza-dC or both inhibitors. (B) Extent of promoter demethylation upon stimulation with PBA or 5-Aza-dC alone or both inhibitors compared with DMSO treated control cells.

5-Aza-2'deoxycytidine and 4-Phenylbutyric acid cause TFF promoter demethylation in retinoblastoma cell lines

To examine the mechanism underlying the changes observed in TFF1 and TFF3 levels following treatment with DNA methyltransferase and histone deacetylase inhibitors, we investigated the methylation status of the TFF promoters in the well characterized retinoblastoma cell line Y-79 following treatment of these cells with 5-Aza-dC, PBA or a combination of both inhibitors. These experiments proved that the induction of TFF1 and TFF3 expression seen in Fig. 5 was accompanied by a reduction in CpG methylation (Fig. 6).

5-Aza-dC alone clearly reduced the number of methylated $\mathrm{CpG}$ sites of the TFF1 promoter, whereas PBA alone only induced minimal changes. Combined treatment further extended the degree of promoter demethylation (70 \%) seen with PBA (4\%) and 5-Aza-dC alone (32\%). Similar effects were found in the TFF3 promoter. Combined treatment strongly increased the extent of demethylation from $5 \%$ (PBA alone) and $25 \%$ (5-Aza-dC alone) to $74 \%$.

Although the same treatment induced similar changes in the methylation status of the TFF2 promoter region no induction of TFF2 expression was detectable (data not shown), strengthening the hypothesis that TFF2 expression is regulated by other mechanisms than promoter methylation in the $\mathrm{Rb}$ cell lines investigated.

\section{Discussion}

\section{Endogenous TFF expression}

In the study presented, we found a clear expression of TFF1 mRNA in all retinoblastoma cell lines analyzed, but only low levels in the healthy human retina (see Fig. 2A). By contrast TFF3 mRNA was clearly expressed in the human retina, but expression was low to not detectable in all retinoblastoma ( $\mathrm{Rb}$ ) cell lines (see Fig. 2C). A similar expression pattern for TFF1 and TFF3 was described for cholangiocarcinoma compared to disease-free control tissues [54].

TFF2, being the only TFF peptide expressed in the murine retina [55], was neither expressed in the human retina nor in any $\mathrm{Rb}$ cell lines investigated (see Fig. 2B). Correspondingly no TFF2 was detected in human conjunctival goblet cells, the cornea, and the nasolacrimal ducts $[7,8,56,57]$. The absence of TFF2 in the human retina might be (i) evolutionary determined and TFF2 expression only be found in the retina of lower vertebrates [50,58], (ii) due to structural differences in the TFF trefoil domains [1], or (iii) based on the absence of another factor (e.g. pRB) required for its expression. 


\section{Correlation of endogenous TFF expression with TFF promoter methylation}

Recent studies indicated that epigenetic mechanisms are also involved in the regulation of TFF expression in cancer. In prostate cancer cell lines promoter hypomethylation of TFF1 and TFF3 has been shown to be closely related to increased expression of these genes [40]. Analyzing the TFF promoter methylation status of Rb cell lines (see Fig. 4), we propose an epigenetic regulation of TFF1 and TFF3 gene expression, where minimal methylation of certain CpGs in the corresponding promoter region results in changes of TFF expression levels (compare Fig. 2A,C). Our data are in good accordance with the finding that as little as 6-8 \% methylation can account for 67-90 \% down-regulation of genes [59]. Along this line, in human breast cancer cell lines a correlation of DNA methylation and TFF1 expression was not observed at all $\mathrm{CpG}$ sites, since some CpGs were unmethylated in TFF1 non-expressing cell lines [60].

In the present study, we could not detect any clear correlation between TFF2 promoter methylation status (see Fig. 4) and TFF2 expression (see Fig. 2B), although hypomethylation of the TFF2 gene was observed in TFF2-overexpressing pancreatic ductal adenocarcinoma [61].

\section{Influence of DNA methylation and histone modification on TFF expression}

Previous studies showed that the DNA methyltransferase inhibitor 5-Aza-dC increases TFF expression in low-expressing prostate cancer cells [40] and restores TFF1 expression in gastric carcinoma cell lines [26]. In our study, we observed a significant induction of TFF3 expression upon stimulation with 5-Aza-dC in all retinoblastoma cell lines (see Fig. 5B) exhibiting no or low endogenous TFF3 expression (compare Fig. 2C). TFF1 expression was, however, only slightly increased by 5-Aza-dC (see Fig. 5A), suggesting a correlation of the extent of up-regulation with endogenous basal expression level, as TFF1 is highly expressed in most of the Rb cell lines analyzed (compare Fig. 2A). No re-expression of TFF2 was observed in Rb cell lines, in which this gene seems to be silenced.

Histone modification is another epigenetic factor playing a key role in transcriptional regulation of gene expression. TFF1 is up-regulated in retinoblastomas with a matching activating histone modification, indicating an epigenetic regulation [45]. A synergistic effect of DNA demethylation and the inhibition of histone deacetylation in the re-expression of silenced genes have been described. In colorectal cancer cells the inhibition of histone deacetylation by trichostatin (TSA) was not sufficient to induce the re-expression of hypermethylated and thereby silenced genes but additional stimulation with 5-Aza-dC resulted in a robust re-expression of silenced cancer-associated genes [62]. Unexpectedly, the results of our study presented here show that treatment with the histone deacetylase inhibitor 4-Phenylbutyric acid (PBA) significantly down-regulated TFF1 expression levels in some Rb cell lines, while up-regulating TFF3 mRNA in other Rb cell lines. Further studies are needed to reveal the mechanism behind this effect, but it might at least be partially explained by different endogenous expression levels of TFF1 and TFF3. Matching results from previous studies, double treatment experiments with 5-Aza-dC further increased TFF1 and TFF3 expression levels in most Rb cell lines investigated (see Fig. 5).

Investigating the promoter methylation after epigenetic drug treatment, we could demonstrate that the TFF1 and TFF3 expression induced by 5-Aza-dC is caused by demethylation of $\mathrm{CpG}$ dinucleotides in the promoter region of the corresponding TFF gene (see Fig. 6). Although 5-Aza-dC leads to a similar demethylation in all TFF promoters (see Fig. 6B), the induction of TFF1 and TFF3 expression highly varies (compare Fig. 5). High induction of TFF3 expression and comparatively slight up-regulation of TFF1 levels support our hypothesis that the extent of up-regulation is somehow dependent on the endogenous TFF expression levels (compare Fig. 2) and indicates that next to epigenetic regulation, TFF1 gene expression might be regulated by additional mechanisms. Treatment with both inhibitors extended the degree of demethylation seen with PBA and 5-Aza-dC alone (see Fig. 6), reflecting the enhanced increase in TFF1 and TFF3 expression levels upon double treatment (compare Fig. 5). 


\section{Involvement of transcription factors in the regulation of TFF expression}

Next to epigenetic regulatory mechanisms, several transcription factors have been found to coordinately regulate TFF expression [17]. Several hypomethylated CpGs in the TFF1 and TFF3 promoter correspond with binding sequences for transcription factors (see Fig. 3), previously shown to be methylation sensitive in a way that $\mathrm{CpG}$ methylation blocks their DNA binding [40]. In the study presented here, we likewise revealed a possible link between the expression level of TFF1 and methylation of CpG -354, matching the binding site for E2F and human ETS1 factor (Elk-1) (see Fig. 3). Methylation of $\geq 60 \%$ at this site (see Fig. 4) corresponds with low TFF1 expression in the duodenum, the retina and in the respective Rb cell lines RB 247 C3, RB 355 and Y-79 (compare Fig. 2).

In TFF3-expressing prostate cancer cells, CpGs closest to the ATG in the TFF3 promoter region are completely free of methylation and were identified as putative binding sites e.g. for the cAMP-responsive element binding protein (CREB) and human ETS1 factor (Elk1) [40]. Promoting these results, our data suggest a correlation between the methylation status of the CREB binding site ( $\mathrm{CpG}-127$ ) in the TFF3 promoter region (see Fig. 3) and TFF3 expression in $\mathrm{Rb}$ cell lines compared to the retina and duodenum reference (compare Fig. 2C). CpG -127 is the only differentially methylated site in the TFF3 promoter region of the human retina and one of the four CpGs with only $40 \%$ methylation in the TFF3 promoter of the duodenum (compare Fig. 4), both displaying high endogenous TFF3 expression (compare Fig. 2C).

\section{Summary}

Summarizing one can state that TFF3 gene expression is inducible by 5-Aza-dC treatment (see Fig. 5) and this induction is accompanied by promoter demethylation (compare Fig. 6), indicating an epigenetic regulation for TFF3 expression in retinoblastoma cell lines. Our results, however, suggest that the regulation of at least TFF1 and TFF2 expression in $\mathrm{Rb}$ cell lines requires the involvement of additional mechanisms other than epigenetic regulation, e.g. binding of transcription factors independent of the methylation status of the TFF promoter.

Although we could demonstrate a correlation between the TFF1 promoter methylation status (see Fig. 4) and the corresponding gene expression in nearly all $\mathrm{Rb}$ cell lines investigated (compare Fig. 2), TFF1 expression was only slightly up-regulated upon 5-Aza$\mathrm{dC}$ treatment (see Fig. 5). In the light of a clear accompanying demethylation in the TFF promoter region of all TFFs in the well characterized retinoblastoma cell line Y-79 (see Fig. 6) and low endogenous TFF1 expression levels (see Fig. 2), one would have expected a high induction of TFF1 expression upon 5-Aza-dC treatment. This also holds true for TFF2 expression, which was not inducible by 5 -Aza-dC, PBA or combined treatment despite obvious changes in the TFF2 promoter methylation status. Ongoing studies investigating the TFF promoter methylation of all $\mathrm{Rb}$ cell lines upon epigenetic drug treatment and correlating the methylation status with the induction of TFF expression in the respective cell line are required to expand our proposed epigenetic regulation model.

\section{Disclosure Statement}

The authors have no conflicts to declare.

\section{Acknowledgments}

The authors would like to thank S. Thanos, E. Cario and R. Doliva for kindly providing human tissue samples. We also would like to thank U. Gerster and U. Laub for excellent technical assistance and D. Gioè for proofreading of the manuscript. 
Philippeit/Busch/Dünker: Epigenetic Regulation of TFFs in Rb Cells

\section{References}

1 Thim L, May FE: Structure of mammalian trefoil factors and functional insights. Cell Mol Life Sci 2005;62:2956-2973.

- Hoffmann W: Trefoil factors tff (trefoil factor family) peptide-triggered signals promoting mucosal restitution. Cell Mol Life Sci 2005;62:2932-2938.

3 Kjellev S: The trefoil factor family - small peptides with multiple functionalities. Cell Mol Life Sci 2009;66:1350-1369.

4 Hirota M, Awatsuji H, Sugihara Y, Miyashita S, Furukawa Y, Hayashi K: Expression of ps2 gene in rat brain. Biochem Mol Biol Int 1995;35:1079-1084.

5 Hoffmann W, Jagla W, Wiede A: Molecular medicine of tff-peptides: From gut to brain. Histol Histopathol 2001;16:319-334.

6 Hoffmann W, Jagla W: Cell type specific expression of secretory tff peptides: Colocalization with mucins and synthesis in the brain. Int Rev Cytol 2002;213:147-181.

7 Hinz M, Schwegler H, Chwieralski CE, Laube G, Linke R, Pohle W, Hoffmann W: Trefoil factor family (tff) expression in the mouse brain and pituitary: Changes in the developing cerebellum. Peptides 2004;25:827832 .

-8 Steven P, Schafer G, Nolle B, Hinz M, Hoffmann W, Paulsen F: Distribution of tff peptides in corneal disease and pterygium. Peptides 2004;25:819-825.

-9 Paulsen FP, Woon CW, Varoga D, Jansen A, Garreis F, Jager K, Amm M, Podolsky DK, Steven P, Barker NP, Sel S: Intestinal trefoil factor/tff3 promotes re-epithelialization of corneal wounds. J Biol Chem 2008;283:13418-13427.

10 Jakowlew SB, Breathnach R, Jeltsch JM, Masiakowski P, Chambon P: Sequence of the ps2 mrna induced by estrogen in the human breast cancer cell line mcf-7. Nucleic Acids Res 1984;12:2861-2878.

11 Nunez AM, Jakowlev S, Briand JP, Gaire M, Krust A, Rio MC, Chambon P: Characterization of the estrogeninduced ps2 protein secreted by the human breast cancer cell line mcf-7. Endocrinology 1987;121:17591765.

12 Bonkhoff H, Stein U, Welter C, Remberger K: Differential expression of the ps2 protein in the human prostate and prostate cancer: Association with premalignant changes and neuroendocrine differentiation. Hum Pathol 1995;26:824-828.

13 May FE, Westley BR: Expression of human intestinal trefoil factor in malignant cells and its regulation by oestrogen in breast cancer cells. J Pathol 1997;182:404-413.

14 Perry JK, Kannan N, Grandison PM, Mitchell MD, Lobie PE: Are trefoil factors oncogenic? Trends Endocrinol Metab 2008;19:74-81.

15 Taupin D, Pedersen J, Familari M, Cook G, Yeomans N, Giraud AS: Augmented intestinal trefoil factor (tff3) and loss of ps2 (tff1) expression precedes metaplastic differentiation of gastric epithelium. Lab Invest 2001;81:397-408.

16 Faith DA, Isaacs WB, Morgan JD, Fedor HL, Hicks JL, Mangold LA, Walsh PC, Partin AW, Platz EA, Luo J, De Marzo AM: Trefoil factor 3 overexpression in prostatic carcinoma: Prognostic importance using tissue microarrays. Prostate 2004;61:215-227.

17 Emami S, Rodrigues S, Rodrigue CM, Le Floch N, Rivat C, Attoub S, Bruyneel E, Gespach C: Trefoil factor family (tff) peptides and cancer progression. Peptides 2004;25:885-898.

18 Leung WK, Yu J, Chan FK, To KF, Chan MW, Ebert MP, Ng EK, Chung SC, Malfertheiner P, Sung JJ: Expression of trefoil peptides (tff1, tff2, and tff3) in gastric carcinomas, intestinal metaplasia, and non-neoplastic gastric tissues. J Pathol 2002;197:582-588.

19 Terris B, Dubois S, Buisine MP, Sauvanet A, Ruszniewski P, Aubert JP, Porchet N, Couvelard A, Degott C, Flejou JF: Mucin gene expression in intraductal papillary-mucinous pancreatic tumours and related lesions. J Pathol 2002;197:632-637.

20 Okada H, Kimura MT, Tan D, Fujiwara K, Igarashi J, Makuuchi M, Hui AM, Tsurumaru M, Nagase H: Frequent trefoil factor 3 (tff3) overexpression and promoter hypomethylation in mouse and human hepatocellular carcinomas. Int J Oncol 2005;26:369-377.

-21 Uchino H, Kataoka H, Itoh H, Hamasuna R, Koono M: Overexpression of intestinal trefoil factor in human colon carcinoma cells reduces cellular growth in vitro and in vivo. Gastroenterology 2000;118:60-69. 
Philippeit/Busch/Dünker: Epigenetic Regulation of TFFs in Rb Cells

22 Poulsom R, Hanby AM, Lalani EN, Hauser F, Hoffmann W, Stamp GW: Intestinal trefoil factor (tff 3) and ps2 (tff 1), but not spasmolytic polypeptide (tff 2) mrnas are co-expressed in normal, hyperplastic, and neoplastic human breast epithelium. J Pathol 1997;183:30-38.

23 Khoury T, Chadha K, Javle M, Donohue K, Levea C, Iyer R, Okada H, Nagase H, Tan D: Expression of intestinal trefoil factor (tff-3) in hepatocellular carcinoma. Int J Gastrointest Cancer 2005;35:171-177.

-24 Yamachika T, Werther JL, Bodian C, Babyatsky M, Tatematsu M, Yamamura Y, Chen A, Itzkowitz S: Intestinal trefoil factor: A marker of poor prognosis in gastric carcinoma. Clin Cancer Res 2002;8:1092-1099.

25 Yio X, Diamond M, Zhang JY, Weinstein H, Wang LH, Werther L, Itzkowitz S: Trefoil factor family-1 mutations enhance gastric cancer cell invasion through distinct signaling pathways. Gastroenterology 2006;130:1696-1706.

26 Feng G, Zhang Y, Yuan H, Bai R, Zheng J, Zhang J, Song M: DNA methylation of trefoil factor 1 (tff1) is associated with the tumorigenesis of gastric carcinoma. Mol Med Rep 2014;9:109-117.

27 Buache E, Etique N, Alpy F, Stoll I, Muckensturm M, Reina-San-Martin B, Chenard MP, Tomasetto C, Rio MC: Deficiency in trefoil factor 1 (tff1) increases tumorigenicity of human breast cancer cells and mammary tumor development in tff1-knockout mice. Oncogene 2011;30:3261-3273.

28 Bird A: The essentials of DNA methylation. Cell 1992;70:5-8.

29 Bird A: DNA methylation patterns and epigenetic memory. Genes Dev 2002;16:6-21.

30 Jones PA, Baylin SB: The fundamental role of epigenetic events in cancer. Nat Rev Genet 2002;3:415-428.

-31 Zhang YW, Staal B, Dykema KJ, Furge KA, Vande Woude GF: Cancer-type regulation of mig- 6 expression by inhibitors of methylation and histone deacetylation. PLoS One 2012;7:e38955.

-32 Bird AP: Cpg-rich islands and the function of DNA methylation. Nature 1986;321:209-213.

-33 Antequera F, Bird A: Number of cpg islands and genes in human and mouse. Proc Natl Acad Sci U S A 1993;90:11995-11999.

-34 Jaenisch R, Bird A: Epigenetic regulation of gene expression: How the genome integrates intrinsic and environmental signals. Nat Genet 2003;33 Suppl:245-254.

35 Eden S, Hashimshony T, Keshet I, Cedar H, Thorne AW: DNA methylation models histone acetylation. Nature 1998;394:842.

-36 Baus-Loncar M, Giraud AS: Multiple regulatory pathways for trefoil factor (tff) genes. Cell Mol Life Sci 2005;62:2921-2931.

37 Tomasetto C, Rockel N, Mattei MG, Fujita R, Rio MC: The gene encoding the human spasmolytic protein (sml1/hsp) is in 21q 22.3, physically linked to the homologous breast cancer marker gene bcei/ps2. Genomics 1992;13:1328-1330.

-38 Chinery R, Williamson J, Poulsom R: The gene encoding human intestinal trefoil factor (tff3) is located on chromosome 21q22.3 clustered with other members of the trefoil peptide family. Genomics 1996;32:281284.

39 Gott P, Beck S, Machado JC, Carneiro F, Schmitt H, Blin N: Human trefoil peptides: Genomic structure in 21q22.3 and coordinated expression. Eur J Hum Genet 1996;4:308-315.

40 Vestergaard EM, Nexo E, Torring N, Borre M, Orntoft TF, Sorensen KD: Promoter hypomethylation and upregulation of trefoil factors in prostate cancer. Int J Cancer 2010;127:1857-1865.

-41 Gardiner-Garden M, Frommer M: Cpg islands in vertebrate genomes. J Mol Biol 1987;196:261-282.

42 Beck S, Sommer P, Blin N, Gott P: 5'-flanking motifs control cell-specific expression of trefoil factor genes (tff). Int J Mol Med 1998;2:353-361.

43 Ribieras S, Lefebvre 0, Tomasetto C, Rio MC: Mouse trefoil factor genes: Genomic organization, sequences and methylation analyses. Gene 2001;266:67-75.

44 Theriault BL, Dimaras H, Gallie BL, Corson TW: The genomic landscape of retinoblastoma: A review. Clin Experiment Ophthalmol 2014;42:33-52.

45 Zhang J, Benavente CA, McEvoy J, Flores-Otero J, Ding L, Chen X, Ulyanov A, Wu G, Wilson M, Wang J, Brennan R, Rusch M, Manning AL, Ma J, Easton J, Shurtleff S, Mullighan C, Pounds S, Mukatira S, Gupta P, Neale G, Zhao D, Lu C, Fulton RS, Fulton LL, Hong X, Dooling DJ, Ochoa K, Naeve C, Dyson NJ, Mardis ER, Bahrami A, Ellison D, Wilson RK, Downing JR, Dyer MA: A novel retinoblastoma therapy from genomic and epigenetic analyses. Nature 2012;481:329-334.

-46 Reid TW, Albert DM, Rabson AS, Russell P, Craft J, Chu EW, Tralka TS, Wilcox JL: Characteristics of an established cell line of retinoblastoma. J Natl Cancer Inst 1974;53:347-360. 
47 McFall RC, Sery TW, Makadon M: Characterization of a new continuous cell line derived from a human retinoblastoma. Cancer Res 1977;37:1003-1010.

48 Gallie BL, Holmes W, Phillips RA: Reproducible growth in tissue culture of retinoblastoma tumor specimens. Cancer Res 1982;42:301-305.

49 Griegel S, Hong C, Frotschl R, Hulser DF, Greger V, Horsthemke B, Rajewsky MF: Newly established human retinoblastoma cell lines exhibit an "immortalized" but not an invasive phenotype in vitro. Int J Cancer 1990;46:125-132.

-50 Weise A, Dunker N: High trefoil factor 1 (tff1) expression in human retinoblastoma cells correlates with low growth kinetics, increased cyclin-dependent kinase (cdk) inhibitor levels and a selective downregulation of cdk6. Histochem Cell Biol 2013;139:323-338.

51 Podolsky DK, Lynch-Devaney K, Stow JL, Oates P, Murgue B, De-Beaumont M, Sands BE, Mahida YR: Identification of human intestinal trefoil factor. Goblet cell-specific expression of a peptide targeted for apical secretion. J Biol Chem 1993;268:12230.

52 Paulsen F, Varoga D, Paulsen A, Tsokos M: Trefoil factor family (tff) peptides of normal human vater's ampulla. Cell Tissue Res 2005;321:67-74.

53 Cartharius K, Frech K, Grote K, Klocke B, Haltmeier M, Klingenhoff A, Frisch M, Bayerlein M, Werner T: Matinspector and beyond: Promoter analysis based on transcription factor binding sites. Bioinformatics 2005;21:2933-2942.

54 Kosriwong K, Menheniott TR, Giraud AS, Jearanaikoon P, Sripa B, Limpaiboon T: Trefoil factors: Tumor progression markers and mitogens via egfr/mapk activation in cholangiocarcinoma. World J Gastroenterol 2011;17:1631-1641.

55 Paunel-Gorgulu AN, Franke AG, Paulsen FP, Dunker N: Trefoil factor family peptide 2 acts pro-proliferative and pro-apoptotic in the murine retina. Histochem Cell Biol 2011;135:461-473.

-56 Langer G, Jagla W, Behrens-Baumann W, Walter S, Hoffmann W: Secretory peptides tff1 and tff3 synthesized in human conjunctival goblet cells. Invest Ophthalmol Vis Sci 1999;40:2220-2224.

57 Paulsen FP, Berry MS: Mucins and tff peptides of the tear film and lacrimal apparatus. Prog Histochem Cytochem 2006;41:1-53.

58 Jiang Z, Lossie AC, Applegate TJ: Evolution of trefoil factor(s): Genetic and spatio-temporal expression of trefoil factor 2 in the chicken (gallus gallus domesticus). PLoS One 2011;6:e22691.

59 Hsieh CL: Dependence of transcriptional repression on cpg methylation density. Mol Cell Biol 1994;14:5487-5494.

60 Martin V, Ribieras S, Song-Wang X, Rio MC, Dante R: Genomic sequencing indicates a correlation between DNA hypomethylation in the 5 ' region of the ps2 gene and its expression in human breast cancer cell lines. Gene 1995;157:261-264.

61 Sato N, Maitra A, Fukushima N, van Heek NT, Matsubayashi H, Iacobuzio-Donahue CA, Rosty C, Goggins M: Frequent hypomethylation of multiple genes overexpressed in pancreatic ductal adenocarcinoma. Cancer Res 2003;63:4158-4166.

62 Cameron EE, Bachman KE, Myohanen S, Herman JG, Baylin SB: Synergy of demethylation and histone deacetylase inhibition in the re-expression of genes silenced in cancer. Nat Genet 1999;21:103-107.

63 Jeltsch JM, Roberts M, Schatz C, Garnier JM, Brown AM, Chambon P: Structure of the human oestrogenresponsive gene ps2. Nucleic Acids Res 1987;15:1401-1414.

64 Beck S, Sommer P, dos Santos Silva E, Blin N, Gott P: Hepatocyte nuclear factor 3 (winged helix domain) activates trefoil factor gene tff1 through a binding motif adjacent to the tataa box. DNA Cell Biol 1999;18:157-164. 